\title{
89. Note on an Ergodic Theorem
}

\author{
By Shigeru Tsurumi \\ Mathematical Institute, Tokyo Metropolitan University, Tokyo \\ (Comm. by Z. Suetuna, M.J.A., June 12, 1954)
}

1. Let $(X, \mathfrak{B}, m)$ be a measure space such that $X$ is a set, $\mathfrak{B}$ is a Borel field of subsets of $X$, and $m$ is a $\sigma$-finite measure defined on $\mathfrak{B}$. A single valued (not necessarily one to one) transformation $T$ of $X$ onto itself is called measurable if both $T$ and its inverse transformation $T^{-1}$ transform every set of $\mathfrak{B}$ to a set of $\mathfrak{B}$. The measurable transformation $T$ is called non-singular (with respect to $m$ ) if $E \in \mathfrak{B}$ and $m(E)=0$ imply $m(T E)=m\left(T^{-1} E\right)=0$, and is called incompressible (with respect to $m$ ) if $E \in \mathfrak{B}$ and $T^{-1} E \supset E$ imply $m\left(T^{-1} E-E\right)=0$. Two measures $\lambda$ and $\mu$ defined on $\mathfrak{B}$ are called equivalent if $E \in \mathfrak{B}$ and $\lambda(E)=0$ imply $\mu(E)=0$ and conversely. A measure $\mu$ on $B$ is said to be invariant under the measurable transformation $T$ (or the measurable transformation $T$ is said to be measure-preserving with respect to $\mu)$ if $\mu\left(T^{-1} E\right)=\mu(E)$ for any set $E$ of $\mathfrak{B}$.

If $T$ is measurable and non-singular, we put $\mathfrak{B}_{1}=\left\{T^{-1} E ; E \in \mathfrak{B}\right\}$. Then, from the Radon-Nikodym theorem, there exists a measurable function $w(x)$ such that

$$
m(T E)=\int_{E} w(x) d m
$$

for every set $E$ of $\mathfrak{B}_{1}$. Let us now put

$$
w_{0}(x)=1, \quad w_{n}(x)=w(x) \cdots w\left(T^{n-1} x\right)
$$

for any point $x$ of $X$ and for $n=1,2, \ldots$. Then we obtain the recurrence formula:

for $i, j=0,1,2, \ldots$.

$$
w_{i+j}(x)=w_{i}\left(T^{j} x\right) \cdot w_{j}(x)
$$

Y. N. Dowker $[1]^{1)}$ has offered the following question concerning the extension of Halmos' ergodic theorem [2] for one to one transformation to the case of a single valued transformation: whether, for a single valued, measurable, non-singular transformation $T$ of $X$ onto itself, the condition that $T$ is incompressible (or some similar condition) yields that, for any measurable function $g(x)$ which is positive almost everywhere, the series $\sum_{i=0}^{\infty} g\left(T^{i} x\right) w_{i}(x)$ diverges almost everywhere?

1) Numbers in square brackets refer to the references at the end of this paper. 
The purpose of this note is to show that even if we assume the existence of a finite invariant measure equivalent to $m$ (which is obviously a stronger assumption than the incompressibility of $T$ ), the above series does not necessarily diverge almost everywhere.

2. In the following, we shall show two examples.

Example 1. Let us consider the collection of the linear intervals $J_{n, t}$ 's and $J_{k}^{\prime}$ 's in the $(s, t)$-plane such that

$$
\begin{aligned}
& J_{1, k}=\left\{(s, k) ; \quad 0 \leqq s<1 / 2^{2 k+1}\right\}, \\
& J_{2, k}=\left\{(s, k) ; \quad 1 / 2^{2 k+1} \leqq s<1 / 2^{2 k}\right\}, \\
& J_{k}=J_{1, k} \cup J_{2, k}
\end{aligned}
$$

for $k=0,1,2, \ldots$. Let $(X, \mathfrak{B}, m)$ be the measure space such that $X$ is the union of all $J_{k}$ 's, $\mathfrak{B}$ is the class of Lebesgue measurable subsets of $X$, and $m$ is the ordinary linear Lebesgue measure on $\mathfrak{B}$. Then $(X, \mathfrak{B}, m)$ is obviously a finite measure space. We now define a transformation $T$ by

$$
\begin{aligned}
& T(s, t)=\left(2^{2 k+1} s, 0\right), \quad \text { if } \quad(s, t) \in J_{1, k}, \\
& =\left(\frac{1}{2}\left[s-\frac{1}{2^{2 k+1}}\right], k+1\right), \quad \text { if } \quad(s, t) \in J_{2, k},
\end{aligned}
$$

for $k=0,1,2, \ldots$.

Then we get

(i) $T$ is a single valued, measurable, and non-singular transformation of $X$ onto itself;

(ii) there exists a finite invariant measure equivalent to $m$;

(iii) the series $\sum_{n=0}^{\infty} w_{n}((s, t))$ converges almost everywhere.

The property (i) follows obviously from the definition of $T$.

Proof of (ii): We put for any set $E$ of $\mathfrak{B}$

$$
\mu(E)=\sum_{k=0}^{\infty} 2^{k} \cdot m\left(E \cap J_{k}\right) .
$$

Then $\mu$ is obviously a measure equivalent to $m$ and

$$
\mu(X)=\sum_{k=0}^{\infty} \mu\left(J_{k}\right)=\sum_{k=0}^{\infty} \frac{1}{2^{k}}=2 .
$$

Next, we shall show that $\mu$ is invariant under T. For any set $E$ of $\mathfrak{B}$, we have that

$$
\begin{aligned}
& T^{-1}\left(E \cap J_{0}\right) \subset \bigcup_{k=0}^{\infty} J_{1, k}, \\
& T^{-1}\left(E \cap J_{k}\right) \subset J_{2, k-1}
\end{aligned}
$$

for $k=1,2, \ldots$, and

$$
\mu\left[T^{-1}\left(E \cap J_{k}\right)\right]=\mu\left(E \cup J_{k}\right)
$$

for $k=0,1,2, \ldots$. Thus we get for any set $E$ of $\mathfrak{B}$ 


$$
\begin{aligned}
\mu\left(T^{-1} E\right) & =\mu\left[T^{-1}\left(\bigcup_{k=0}^{\infty}\left(E \cap J_{k}\right)\right)\right]=\sum_{k=0}^{\infty} \mu\left[T^{-1}\left(E \cup J_{k}\right)\right] \\
& =\sum_{k=0}^{\infty} \mu\left(E \cap J_{k}\right)=\mu(E),
\end{aligned}
$$

which is the required.

Proof of (iii): From the definition of $w$, it is easy to see that

$$
\begin{aligned}
w((s, t)) & =\frac{3}{2}, \quad \text { if } \quad(s, t) \in \bigcup_{k=0}^{\infty} J_{1, k}, \\
& =\frac{1}{2}, \quad \text { if } \quad(s, t) \in \bigcup_{k=0}^{\infty} ! J_{2, k} .
\end{aligned}
$$

We shall now define a new single valued transformation $\varphi$ of $J_{0}$ onto itself by

$$
\varphi((s, 0))=(\{2 s\}, 0),
$$

where $\{x\}$ denotes the fractional part of $x$. Then we have, for any point $(s, 0)$ of $J_{0}$ and $n=0,1,2, \ldots$,

$$
\begin{aligned}
& w\left(T^{n}(s, 0)\right)=w\left(\phi^{n}(s, 0)\right), \\
w_{n}((s, 0))= & w((s, 0)) \cdot w(T(s, 0)) \cdots w\left(T^{n-1}(s, 0)\right) \\
= & w((s, 0)) \cdot w(\varphi(s, 0)) \cdots w\left(\varphi^{n-1}(s, 0)\right) .
\end{aligned}
$$

Hence, let $f$ be the characteristic function of $J_{1,0}$, then we have by Raikov's theorem [3] that for almost all points $(s, 0)$ of $J_{0}$

$$
\lim _{n \rightarrow \infty} 1 \sum_{i=0}^{n-1} f\left(\varphi^{i}(s, 0)\right)=\int_{J_{0}} f d m=m\left(J_{1,0}\right)=\frac{1}{2} \text {. }
$$

Now we have for any point $(s, 0)$ of $J_{0}$

$$
\sum_{i=0}^{n-1} f\left(\phi^{i}(s, 0)\right)
$$

$=\left[\right.$ Number of elements $i$ 's such that $\varphi^{i}(s, 0) \in J_{1,0}$ for $\left.0 \leqq i \leqq n-1\right]$

$=\left[N u m b e r\right.$ of elements $i$ 's such that $w\left(\phi^{i}(s, 0)\right)=3 / 2$ for $\left.0 \leqq i \leqq n-1\right]$, and further, for any point $(s, 0)$ of $J_{2,0}$ and $i=0,1,2, \ldots$,

$$
w\left(\varphi^{i}(s, 0)\right)=\frac{1}{2} \text {. }
$$

Thus, from (1), (2) and (3), it follows that for almost all points $(s, 0)$ of $J_{0}$

$$
w_{n}((s, 0))=O\left(\left(\begin{array}{ll}
3 & 1 \\
2 & 2
\end{array}\right)^{n}\right)=O\left(\left(\frac{3}{4}\right)^{n}\right)
$$

so that

$$
\sum_{n=0}^{\infty} w_{n}((s, 0))<\infty .
$$

On the other hand, for any point $(s, t)$ of $X$, there exists a positive integer $p(s, t)$ such that 


$$
T^{p s s, t)}(s, t) \in J_{0} .
$$

Then we have by the recurrence formula that for any point $(s, t)$ of $X$

$$
\begin{aligned}
& \sum_{n=0}^{\infty} w_{n}((s, t))=\sum_{n=0}^{p(s, t)-1} w_{n}((s, t))+\sum_{n=0}^{\infty} w_{n+p(s, t)}((s, t)) \\
& =\sum_{n=0}^{p(s, t)-1} w_{n}((s, t))+\sum_{n=0}^{\infty} w_{n}\left(T^{p(s, t)}(s, t)\right) \cdot w_{p(s, t)}((s, t)) .
\end{aligned}
$$

Thus, from (4), (5) and (6), we get

$$
\sum_{n=0}^{\infty} w_{n}((s, t))<\infty
$$

almost everywhere on $X$.

Remark. We notice that, in case $m$ is finite as in Example 1, the series $\sum_{n=0}^{\infty} w_{n}(x)$ cannot be uniformly bounded. In fact, if we assume that the series $\sum_{n=0}^{\infty} w_{n}(x)$ is uniformly bounded and $K$ is its majorant, then we have

$$
\begin{aligned}
\infty>K \cdot m(X) & \geqq \int_{X} \sum_{n=0}^{\infty} w_{n}(x) d m=\sum_{n=0}^{\infty} \int_{X} w_{n}(x) d m \\
& =\sum_{n=0}^{\infty} \int_{T^{-n} X_{X}} w_{n}(x) d m=\sum_{n=0}^{\infty} m(X)=\infty,
\end{aligned}
$$

which is a contradiction.

However it will be shown in the following example that the above series may be uniformly bounded for some $\sigma$-finite (not finite) measure space and some transformation.

Example 2. Let us consider the collection of the linear intervals $J_{n, k}$ 's and $J_{k}^{\prime}$ 's in the $(s, t)$-plane such that

$$
\begin{aligned}
& J_{1, k}=\left\{(s, k) ; \quad 0 \leqq s<2^{k-2}\right\}, \\
& J_{2, k}=\left\{(s, k) ; \quad 2^{k-2} \leqq s<2^{k-2}+2^{k-1}\right\}, \\
& J_{1,-k}=\left\{(s,-k) ; \quad 0 \leqq s<1 / 2^{2 k}\right\} \\
& J_{2,-k}=\left\{(s,-k) ; \quad 1 / 2^{2 k} \leqq s<1 / 2^{2 k}+1 / 2^{2 k+1}\right\}
\end{aligned}
$$

for $k=1,2, \ldots$, and

$$
\begin{aligned}
& J_{1,0}=\{(s, 0) ; \quad 0 \leqq s<1 / 2\}, \\
& J_{2,0}=\{(s, 0) ; \quad 1 / 2 \leqq s<1 / 2+1 / 2\},
\end{aligned}
$$

and

$$
J_{k}=J_{1, k} \cup J_{2, k}
$$

for $k=0, \pm 1, \pm 2, \ldots$. Let $(X, \mathfrak{B}, m)$ be the measure space such that $X$ is the union of all $J_{k}$ 's, $\mathfrak{B}$ is the class of Lebesgue measurable subsets of $X$, and $m$ is the ordinary linear Lebesgue measure. Then $(X, \mathfrak{B}, m)$ is a $\sigma$-finite (not finite) measure space. We now define a transformation $T$ by 


$$
\begin{array}{rlrl}
T(s, t) & =(6 s, k+1), & & \text { if }(s, t) \in J_{1, k} \text { for } k=1,2, \ldots, \\
& =\left(\frac{3}{4}\left[s-2^{k-2}\right], k-1\right), & & \text { if }(s, t) \in J_{2, k} \text { for } k=2,3, \ldots, \\
& =\left(s-\frac{1}{2}, 0\right), & & \text { if }(s, t) \in J_{2,1}, \\
& =(3 s, 1), & & \text { if }(s, t) \in J_{1,0}, \\
& \left.=\left(\frac{3}{4}[s-1] 2\right],-1\right), & & \text { if }(s, t) \in J_{2,0}, \\
& =(4 s, 0), & & \text { if }(s, t) \in J_{1,-1}, \\
& =(6 s,-k+1), & & \text { if }(s, t) \in J_{1,-k} \text { for } k=2,3, \ldots, \\
& =\left(\frac{3}{4}[s-1\right. \\
\left.\left.2^{2 \bar{k}}\right],-k-1\right), & \text { if }(s, t) \in J_{2,-k} \text { for } k=1,2, \ldots .
\end{array}
$$

Then we get

(i) $T$ is a single valued, measurable, and non-singular transformation of $X$ onto itself;

(ii) there exists a finite invariant measure equivalent to $m$;

(iii) the series $\sum_{n=0}^{\infty} w_{n}((s, t))$ is uniformly bounded on $X$.

The proof of (i) follows directly from the definition of $T$.

Proof of (ii): We put for any set $E$ of $\mathfrak{B}$

$$
\mu(E)=\sum_{k=1}^{\infty} \frac{1}{2^{2 k-1}} \cdot m\left(E \cap J_{k}\right)+m\left(E \cap J_{0}\right)+\sum_{k=1}^{\infty} 2^{k} \cdot m\left(E \cap J_{-k}\right) .
$$

Then it can be proved similarly as Example 1 that $\mu$ is a finite invariant measure on $\mathfrak{B}$ equivalent to $m$.

Proof of (iii): From the definition of $w$, it is easy to see that

$$
\begin{aligned}
w((s, t)) & =\frac{2}{3}, \quad \text { if }(s, t) \in \bigcup_{k=1}^{\infty} J_{1, k} \cup \bigcup_{k=3}^{\infty} J_{2, k} \cup \bigcup_{k=2}^{\infty} J_{1,-k} \cup \bigcup_{k=0}^{\infty} J_{2,-k}, \\
& =\frac{3}{5}, \quad \text { if }(s, t) \in J_{2,2} \cup J_{1,0}, \\
& =\frac{4}{5}, \quad \text { if }(s, t) \in J_{2,1} \cup J_{1,-1} .
\end{aligned}
$$

Thus we have

$$
\sum_{n=0}^{\infty} w_{n}((s, t))<1+\sum_{n=1}^{\infty}\left(\begin{array}{c}
4 \\
5
\end{array}\right)^{n}=5
$$

almost everywhere on $X$.

\section{References}

[1] Y. N. Dowker: A new proof of the general ergodic theorem, Acta Szeged., 12, 162-166 (1952).

[2] P. R. Halmos: An ergodic theorem, Proc. Nat. Acad. Sci. U.S.A., 32, 156161 (1946).

[3] D. Raikov: On some arithmetical properties of summable functions, Rec. Math., nouvelle série, 1, 377-384 (1936). 